\title{
Discontinuity of the chemical potential in reduced-density-matrix-functional theory for open-shell systems
}

\author{
N. Helbig, ${ }^{1,2,3}$ N. N. Lathiotakis, ${ }^{1,3,4}$ and E. K. U. Gross ${ }^{1,3}$ \\ ${ }^{1}$ Institut für Theoretische Physik, Freie Universität Berlin, Arnimallee 14, D-14195 Berlin, Germany \\ ${ }^{2}$ Unité de Physico-Chimie et de Physique des Matériaux, Université Catholique de Louvain, B-1348 Louvain-la-Neuve, Belgium \\ ${ }^{3}$ European Theoretical Spectroscopy Facility \\ ${ }^{4}$ Theoretical and Physical Chemistry Institute, The National Hellenic Research Foundation, Vass. Constantinou 48, 11635 Athens, Greece
}

(Received 18 March 2008; published 6 February 2009)

\begin{abstract}
We employ reduced-density-matrix-functional theory in the calculation of the fundamental gap of open-shell systems. The formula for the calculation of the fundamental gap is derived with special attention to the spins of the neutral and the charged systems. We discuss the effects of different functionals as well as the changes due to different basis sets. Also, we investigate the importance of varying the natural orbitals for the calculation of the fundamental gap.
\end{abstract}

DOI: $10.1103 /$ PhysRevA.79.022504

PACS number(s): 31.15.E-, 31.10.+z, 71.10.-w

\section{INTRODUCTION}

Density-functional theory (DFT) $[1,2]$ is a powerful tool to calculate the electronic structure of atoms, molecules, and solids. Within DFT, observables are given as functionals of the particle density. In reduced-density-matrix-functional theory (RDMFT) the one-body reduced density matrix (1$\mathrm{RDM}$ ) is used as the basic variable. RDMFT is based on Gilbert's theorem [3] which proves that each ground-state observable can, in principle, be written as a functional of the 1-RDM. First-generation functionals [4-6] perform very well in the description of the dissociation of small molecules. Second-generation functionals were introduced recently [7-10], showing improved performance both for small molecules [7-11] and for the homogeneous electron gas [12].

A key quantity in electronic structure calculations is the band gap for semiconductors and insulators. It is defined as the difference between the ionization potential $I$ and the electron affinity $A$,

$$
\Delta=I-A,
$$

where

$$
\begin{aligned}
& I=E_{\mathrm{tot}}(N-1)-E_{\mathrm{tot}}(N), \\
& A=E_{\mathrm{tot}}(N)-E_{\mathrm{tot}}(N+1) .
\end{aligned}
$$

$E_{\text {tot }}(N)$ denotes the ground-state energy of an $N$-electron system. In the chemistry literature, $\Delta / 2$ is called the chemical hardness if the system is finite. For simplicity, we use the term fundamental gap for both finite and extended systems throughout this paper. We wish to point out that the fundamental gap differs from what is known as the optical gap. The optical gap is given as the energy necessary to excite the system from the ground state to the first excited state. Therefore, its size is reduced by the binding energy of the created exciton compared to the fundamental gap.

Within DFT, it can be shown $[13,14]$ that the fundamental gap is exactly given by

$$
\Delta=\Delta_{\mathrm{KS}}+\Delta_{\mathrm{xc}}
$$

where $\Delta_{\mathrm{KS}}$ is the energy difference between the lowest unoccupied and the highest occupied Kohn-Sham states and $\Delta_{\mathrm{xc}}$ is the discontinuity of the exchange-correlation potential upon adding and subtracting a fractional charge. This discontinuity is zero for the local density approximation (LDA) and generalized gradient approximations (GGAs), so $\Delta_{\mathrm{KS}}$ is the prediction for the gap within these approximations. However, this prediction deviates strongly from the experimental values. For semiconductors the calculated gap underestimates the experimental value by typically $50 \%$. In extreme cases, such as germanium, the gap vanishes within the LDA. Interestingly, $\Delta_{\mathrm{KS}}$ for the exact exchange functional is very close to the experimental gap for several systems $[15,16]$. Unfortunately, in the case of exact exchange $\Delta_{\mathrm{xc}}$ is not zero and, in fact, was found to be much larger than $\Delta_{\mathrm{KS}}$. Thus, if properly calculated, the band gaps within exact exchange are highly overestimated compared to the experimental values [15-18]. Exact exchange combined with random-phase approximation correlation was recently shown to yield results very close to the experimental values for $\mathrm{Si}, \mathrm{LiF}$, and solid Ar [18] (provided the discontinuity $\Delta_{\mathrm{xc}}$ is properly included). Finally, a recently introduced hybrid functional $[19,20]$ is reported to give gaps in satisfactory agreement with experimental values for a set of 40 simple and binary semiconductors [21]. In particular, germanium is predicted a semiconductor with a gap of $0.56 \mathrm{eV}$.

An alternative formula to (4), for the fundamental gap in DFT, reads [22]

$$
\Delta=\lim _{\eta \rightarrow 0^{+}}[\mu(N+\eta)-\mu(N-\eta)],
$$

where $\mu$ is the chemical potential, and $N$ is the particle number of the system. As Eq. (5) suggests, the chemical potential has a discontinuity at integer particle number $N$. In a recent paper [23], we presented the analogous equation within reduced-density-matrix-functional theory. In particular, we proved that the Lagrange multiplier used to enforce the conservation of particle number is equal to the chemical potential. This theoretical development was applied to small finite 
and prototype periodic systems with very promising results. We wish to emphasize that the analogy between DFT and RDMFT is not at all trivial because of the $N$-representability condition in RDMFT. The occupation numbers are restricted to the interval $[0,1]$ which leads to border minima. For this reason, the generalization of the proof of Eq. (5) from DFT to RDMFT is not straightforward.

In the present work, we deduce a relationship similar to Eq. (5) for open-shell systems. The difficulty in generalizing Eq. (5) to the open-shell case arises from the fact that adding (subtracting) a spin-up electron to (from) an open-shell ground state is not equivalent to adding (subtracting) a spindown electron. Open-shell systems were recently addressed in Ref. [24], where it was demonstrated that it is reasonable to introduce two Lagrange multipliers to keep the number of electrons in each spin channel fixed seperately. An alternative description of open-shell systems was introduced by Leiva and Piris [25]. In that description, however, spin-up and spin-down occupations are equal for all orbitals except the open-shell ones which are fully occupied by the majority spin. The Lagrange multiplier is then spin independent. Here, we employ the treatment suggested in Ref. [24], where each of the two Lagrange multipliers is a function of the two particle numbers corresponding to the two spin components. In the present work, these particle numbers are assumed to be fractional. We show that a proper extension of Eq. (5) is possible, with the resulting equation involving the discontinuities of both Lagrange multipliers. The derivation is presented in Sec. II. Section III contains results for a set of open-shell atoms and a comparison of the closed- and openshell treatments for systems where the neutral system is actually a closed shell. We also investigate the performance of different functionals in the calculation of the fundamental gap.

\section{THE FUNDAMENTAL GAP IN RDMFT}

Reduced-density-matrix-functional theory uses the onebody reduced density matrix

$$
\gamma\left(\mathbf{x}, \mathbf{x}^{\prime}\right)=N \int d \mathbf{x}_{2} \cdots d \mathbf{x}_{N} \Psi^{*}\left(\mathbf{x}^{\prime}, \mathbf{x}_{2}, \ldots, \mathbf{x}_{N}\right) \Psi\left(\mathbf{x}, \mathbf{x}_{2}, \ldots, \mathbf{x}_{N}\right),
$$

where $\Psi$ denotes the many-body wave function and $\mathbf{x}$ $=(\mathbf{r}, \sigma)$. Integration over $d \mathbf{x}$ means integration over space and summation over spin. Throughout this paper, we restrict ourselves, for simplicity, to the "collinear" case, where $\gamma\left(\mathbf{x}, \mathbf{x}^{\prime}\right)=\gamma\left(\mathbf{r} \sigma, \mathbf{r}^{\prime} \sigma^{\prime}\right)$ is diagonal in spin space, i.e.,

$$
\gamma\left(\mathbf{r} \sigma, \mathbf{r}^{\prime} \sigma^{\prime}\right)=\delta_{\sigma \sigma^{\prime}} \gamma^{\sigma}\left(\mathbf{r}, \mathbf{r}^{\prime}\right)
$$

By diagonalizing $\gamma^{\sigma}\left(\mathbf{r}, \mathbf{r}^{\prime}\right)$ one obtains the natural orbitals $\varphi_{j \sigma}$ and the occupation numbers $n_{j \sigma}$, i.e.,

$$
\gamma^{\sigma}\left(\mathbf{r}, \mathbf{r}^{\prime}\right)=\sum_{j=1}^{\infty} n_{j \sigma} \varphi_{j \sigma}^{*}\left(\mathbf{r}^{\prime}\right) \varphi_{j \sigma}(\mathbf{r})
$$

To ensure the $N$-representability of $\gamma$, the occupation numbers are restricted to the interval $[0,1]$ and sum up to the total number of particles, $N$. In closed-shell systems the two spin directions are identical, i.e.,

$$
\begin{aligned}
& n_{j \uparrow}=n_{j \downarrow}, \\
& \varphi_{j \uparrow}=\varphi_{j \downarrow} .
\end{aligned}
$$

Within the spin-dependent formalism, one can define spin-dependent electron affinities and ionization potentials by adding or removing an electron with specific spin,

$$
\begin{aligned}
& I^{\sigma}=E_{\mathrm{tot}}\left(N^{\sigma}-1, N^{\bar{\sigma}}\right)-E_{\mathrm{tot}}\left(N^{\sigma}, N^{\bar{\sigma}}\right), \\
& A^{\sigma}=E_{\mathrm{tot}}\left(N^{\sigma}, N^{\bar{\sigma}}\right)-E_{\mathrm{tot}}\left(N^{\sigma}+1, N^{\bar{\sigma}}\right) .
\end{aligned}
$$

Here, $E_{\mathrm{tot}}\left(N^{\sigma}, N^{\bar{\sigma}}\right)$ representes the ground-state energy of a system with $N=N^{\sigma}+N^{\bar{\sigma}}$ electrons, where $N^{\sigma}$ is the number of electrons with spin $\sigma$ and $N^{\bar{\sigma}}$ is the number of electrons with the opposite spin, $\bar{\sigma}$. Consequently, the ionization potential and electron affinity defined in Eq. (3) are given by

$$
\begin{gathered}
I=\min _{\sigma}\left\{I^{\uparrow}, I^{\downarrow}\right\}, \\
A=\max _{\sigma}\left\{A^{\uparrow}, A^{\downarrow}\right\},
\end{gathered}
$$

i.e., they are respectively the smallest necessary energy for taking away an electron and the maximum energy gained by adding an electron to the neutral system. The fundamental gap then reads

$$
\Delta=\min _{\sigma}\left\{I^{\uparrow}, I^{\downarrow}\right\}-\max _{\sigma}\left\{A^{\uparrow}, A^{\downarrow}\right\} .
$$

In order to derive a formula analogous to Eq. (5) for the fundamental gap (1) within RDMFT, we follow the same path as in DFT $[14,26,27]$ and extend the definition of the total-energy functional $E_{\text {tot }}[\gamma]$ to systems with fractional particle number $M$. Throughout this paper, we use the convention that $N$ denotes an integer number of particles and $M$ a fractional. Such systems can be described as an ensemble consisting of an $N$ - and an $(N+1)$-particle state for $N \leqslant M$ $\leqslant N+1$. Let $\Psi_{N^{\sigma}, N^{\bar{\sigma}}}$ denote an $N$-particle wave function with $N=N^{\sigma}+N^{\bar{\sigma}}$, where, as before, $N^{\sigma}$ is the number of electrons with spin $\sigma$ and $N^{\bar{\sigma}}$ the number of particles with the opposite spin, $\bar{\sigma}$. We consider an ensemble where, compared to the charge-neutral $\left(N^{\sigma}, N^{\bar{\sigma}}\right)$ system, the number of spin- $\sigma$ particles is increased by $\eta^{\sigma}$. The statistical operator describing such an ensemble is given by

$$
\begin{aligned}
\hat{D}_{N^{\sigma}+\eta^{\sigma}, N^{\bar{\sigma}}}= & \left(1-\eta^{\sigma}\right)\left|\Psi_{N^{\sigma}, N^{\bar{\sigma}}}\right\rangle\left\langle\Psi_{N^{\sigma}, N^{\bar{\sigma}}}\right| \\
& +\eta^{\sigma}\left|\Psi_{N^{\sigma_{+}+N^{\bar{\sigma}}}}\right\rangle\left\langle\Psi_{N^{\sigma_{+}+N \bar{\sigma}}}\right|
\end{aligned}
$$

The expectation value of an operator $\hat{O}$ is then given by

$$
O=\operatorname{tr}\left(\hat{D}_{N^{\sigma}+\eta^{\sigma}, N^{\bar{\sigma}}} \hat{O}\right) .
$$

In particular, for $\hat{O}=\hat{\gamma}^{\sigma_{1}}\left(\mathbf{r}, \mathbf{r}^{\prime}\right)$, i.e., the operator representing the $1-\mathrm{RDM}$ of spin- $\sigma_{1}$ particles, we obtain 


$$
\gamma_{N^{\sigma}+\eta^{\sigma}, N^{\bar{\sigma}}}^{\sigma_{1}}\left(\mathbf{r}, \mathbf{r}^{\prime}\right)=\left(1-\eta^{\sigma}\right) \gamma_{N^{\sigma}, N^{\sigma}}^{\sigma_{1}}\left(\mathbf{r}, \mathbf{r}^{\prime}\right)+\eta^{\sigma} \gamma_{N^{\sigma}+1, N^{\sigma}}^{\sigma_{1}}\left(\mathbf{r}, \mathbf{r}^{\prime}\right)
$$

and for $\hat{O}=\hat{H}$, i.e., the Hamiltonian, we get the total ensemble energy

$$
E_{\mathrm{tot}}\left(N^{\sigma}+\eta^{\sigma}, N^{\bar{\sigma}}\right)=\left(1-\eta^{\sigma}\right) E_{\mathrm{tot}}\left(N^{\sigma}, N^{\bar{\sigma}}\right)+\eta^{\sigma} E_{\mathrm{tot}}\left(N^{\sigma}+1, N^{\bar{\sigma}}\right) .
$$

We note, in passing, that the ensemble weights in Eq. (16) are such that the correct normalization of spin-up and spindown densities is achieved, i.e.,

$$
\begin{gathered}
\int d^{3} r \gamma_{N^{\sigma}+\eta^{\sigma}, N^{\bar{\sigma}}}^{\sigma}(\mathbf{r}, \mathbf{r})=N^{\sigma}+\eta^{\sigma}, \\
\int d^{3} r \gamma_{N^{\sigma}+\eta^{\sigma}, N^{\bar{\sigma}}}^{\bar{\sigma}}(\mathbf{r}, \mathbf{r})=N^{\bar{\sigma}} .
\end{gathered}
$$

Reformulating (19), one obtains

$$
\begin{aligned}
E_{\mathrm{tot}}\left(M^{\sigma}, N^{\bar{\sigma}}\right)= & E_{\mathrm{tot}}\left(N^{\sigma}, N^{\bar{\sigma}}\right)+\eta^{\sigma}\left[E_{\mathrm{tot}}\left(N^{\sigma}+1, N^{\bar{\sigma}}\right)\right. \\
& \left.-E_{\mathrm{tot}}\left(N^{\sigma}, N^{\bar{\sigma}}\right)\right]
\end{aligned}
$$

for $N^{\sigma}<M^{\sigma}=N^{\sigma}+\eta^{\sigma}<N^{\sigma}+1$. In analogy, for $N^{\sigma}-1<M^{\sigma}$ $=N^{\sigma}-1+\eta^{\sigma}<N^{\sigma}$ the total energy is given by

$$
\begin{aligned}
E_{\mathrm{tot}}\left(M^{\sigma}, N^{\bar{\sigma}}\right)= & E_{\mathrm{tot}}\left(N^{\sigma}-1, N^{\bar{\sigma}}\right)+\eta^{\sigma}\left[E_{\mathrm{tot}}\left(N^{\sigma}, N^{\bar{\sigma}}\right)\right. \\
& \left.-E_{\mathrm{tot}}\left(N^{\sigma}-1, N^{\bar{\sigma}}\right)\right] .
\end{aligned}
$$

In other words, the total energy depends linearly on $\eta^{\sigma}$ with slope $-A^{\sigma}$ for $N^{\sigma} \leqslant M^{\sigma} \leqslant N^{\sigma}+1$ and slope $-I^{\sigma}$ for $N^{\sigma}-1$ $\leqslant M^{\sigma} \leqslant N^{\sigma}$. Since $A^{\sigma}$ and $I^{\sigma}$ are in general not the same, the derivative $\partial E_{\mathrm{tot}}\left(M^{\sigma}, N^{\bar{\sigma}}\right) / \partial M^{\sigma}$ has a discontinuity at integer particle number $N^{\sigma}$. From Eqs. (11)-(15), one can conclude that the fundamental gap is given by

$$
\begin{aligned}
\Delta= & \min _{\sigma}\left\{\left.\lim _{\eta^{\sigma} \rightarrow 0^{+}} \frac{\partial E_{\mathrm{tot}}\left(M^{\uparrow}, M^{\downarrow}\right)}{\partial M^{\sigma}}\right|_{N^{\sigma}+\eta^{\sigma}, N^{\bar{\sigma}}}\right\} \\
& -\max _{\sigma}\left\{\left.\lim _{\eta^{\sigma} \rightarrow 0^{+}} \frac{\partial E_{\mathrm{tot}}\left(M^{\uparrow}, M^{\downarrow}\right)}{\partial M^{\sigma}}\right|_{N^{\sigma}-\eta^{\sigma}, N^{\bar{\sigma}}}\right\} .
\end{aligned}
$$

In Ref. [24], we argued that, for open-shell systems, the following functional should be minimized:

$$
F[\gamma]=E_{\text {tot }}[\gamma]-\mu^{\uparrow}\left(\sum_{j=1}^{\infty} n_{j \uparrow}-M^{\uparrow}\right)-\mu^{\downarrow}\left(\sum_{j=1}^{\infty} n_{j \downarrow}-M^{\downarrow}\right) .
$$

The Lagrange multipliers $\mu^{\uparrow}$ and $\mu^{\downarrow}$ are introduced to achieve given particle numbers $M^{\uparrow}$ and $M^{\downarrow}$. To prove the formula for the fundamental gap we first show that these Lagrange multipliers are nothing but the chemical potentials, i.e.,

$$
\mu^{\sigma}\left(M_{1}^{\uparrow}, M_{1}^{\downarrow}\right)=\left.\frac{\partial E_{\text {tot }}\left(M^{\uparrow}, M^{\downarrow}\right)}{\partial M^{\sigma}}\right|_{M_{1}^{\uparrow}, M_{1}^{\downarrow}} .
$$

The derivation of this formula differs significantly from the derivation of its counterpart in DFT due to the abovementioned $N$-representability constraint. In order for the 1-RDM to be connected to an antisymmetric $N$-particle wave function, its occupation numbers have to be restricted to the interval $[0,1][28]$. One can show that the same constraint ensures ensemble $N$-representability for fractional particle number. As a result of this additional constraint, $\delta F / \delta \gamma$ need not vanish at the minimum energy. It is possible that certain occupation numbers are pinned at the border of the interval while the true minimum is obtained for values of $n_{j \sigma}$ outside this interval. The functional $F$ then has a border minimum, and therefore nonvanishing derivative, in all directions where occupation numbers are pinned at zero or one.

We investigate the total energy difference

$$
\begin{aligned}
\delta E_{\mathrm{tot}} & =E_{\mathrm{tot}}\left(M^{\sigma}+\eta^{\sigma}, M^{\bar{\sigma}}\right)-E_{\mathrm{tot}}\left(M^{\sigma}, M^{\bar{\sigma}}\right) \\
& =E\left[\gamma_{M^{\sigma}+\eta^{\sigma}, M^{\bar{\sigma}}}\right]-E\left[\gamma_{M^{\sigma}, M^{\bar{\sigma}}}\right] .
\end{aligned}
$$

As the total energy is a functional of the occupation numbers and natural orbitals, this energy difference is given by

$$
\begin{aligned}
\delta E_{\text {tot }}= & \sum_{\sigma_{1}=\uparrow \downarrow} \sum_{j=1}^{\infty} \int d^{3} r\left(\frac{\delta E_{\text {tot }}}{\delta \varphi_{j \sigma_{1}}(\mathbf{r})} \delta \varphi_{j \sigma_{1}}(\mathbf{r})\right. \\
& \left.+\frac{\delta E_{\text {tot }}}{\delta \varphi_{j \sigma_{1}}^{*}(\mathbf{r})} \delta \varphi_{j \sigma_{1}}^{*}(\mathbf{r})\right)+\sum_{\sigma_{1}=\uparrow \downarrow} \sum_{j=1}^{\infty} \frac{\delta E_{\text {tot }}}{\delta n_{j \sigma_{1}}} \delta n_{j \sigma_{1}} .
\end{aligned}
$$

Using Eq. (25) we can rewrite this change in the total energy as

$$
\begin{aligned}
\delta E_{\mathrm{tot}}= & \sum_{\sigma_{1}=\uparrow \downarrow} \sum_{j=1}^{\infty} \int d^{3} r\left(\frac{\delta F}{\delta \varphi_{j \sigma_{1}}(\mathbf{r})} \delta \varphi_{j \sigma_{1}}(\mathbf{r})\right. \\
& \left.+\frac{\delta F}{\delta \varphi_{j \sigma_{1}}^{*}(\mathbf{r})} \delta \varphi_{j \sigma_{1}}^{*}(\mathbf{r})\right)+\sum_{\sigma_{1}=\uparrow \downarrow} \sum_{j=1}^{\infty}\left(\frac{\delta F}{\delta n_{j \sigma_{1}}}+\mu^{\sigma_{1}}\right) \delta n_{j \sigma_{1}} .
\end{aligned}
$$

At the solution point, the variation with respect to the natural orbitals vanishes such that the first term on the right is zero. The variation with respect to the occupation numbers, however, need not vanish due to the $N$-representability constraint. The sum over all changes $\delta n_{j \sigma_{1}}$ has to give $\eta^{\sigma}$ for the spin channel whose particle number is changed and zero for the other spin channel in order for the new occupation numbers to sum up to the correct particle numbers. Hence, we obtain

$$
\begin{gathered}
E_{\mathrm{tot}}\left(M^{\sigma}+\eta^{\sigma}, M^{\bar{\sigma}}\right)-E_{\mathrm{tot}}\left(M^{\sigma}, M^{\bar{\sigma}}\right) \\
\quad=\mu^{\sigma} \eta^{\sigma}+\sum_{\sigma_{1}=\uparrow \downarrow} \sum_{p} \frac{\delta F}{\delta n_{p \sigma_{1}}} \delta n_{p \sigma_{1}} .
\end{gathered}
$$

Finally, we discuss the contribution of the pinned states. As stated before, for these states $\delta F / \delta n_{p}$ is different from zero and the true minimum of the functional lies outside the in- 
terval $[0,1]$. More specifically, it lies at a finite distance from the border of the interval such that the addition or subtraction of an infinitesimal fraction $\eta^{\sigma}$ of a particle cannot move the minimum into the interval. Therefore, these particle numbers remain pinned upon adding or subtracting an infinitesimal $\eta^{\sigma}$, i.e., $\delta n_{p \sigma_{1}}$ is zero in the limit $\eta^{\sigma} \rightarrow 0$. We, therefore, conclude that

$$
\mu^{\sigma}\left(M_{1}^{\uparrow}, M_{1}^{\downarrow}\right)=\left.\lim _{\eta^{\sigma} \rightarrow 0^{+}}\left(\frac{E_{\mathrm{tot}}\left(M^{\sigma}+\eta^{\sigma}, M^{\bar{\sigma}}\right)-E_{\mathrm{tot}}\left(M^{\sigma}, M^{\bar{\sigma}}\right)}{\eta^{\sigma}}\right)\right|_{M_{1}^{\uparrow}, M_{1}^{\downarrow}}=\left.\frac{\partial E_{\mathrm{tot}}\left(M^{\uparrow}, M^{\downarrow}\right)}{\partial M^{\sigma}}\right|_{M_{1}^{\uparrow}, M_{1}^{\downarrow}}
$$

Using Eq. (24) we obtain the final result for the fundamental gap,

$$
\begin{aligned}
\Delta= & \min _{\sigma}\left(\lim _{\eta^{\sigma} \rightarrow 0^{+}} \mu^{\sigma}\left(M^{\sigma}+\eta^{\sigma}, M^{\bar{\sigma}}\right)\right) \\
& -\max _{\sigma}\left(\lim _{\eta^{\sigma} \rightarrow 0^{+}} \mu^{\sigma}\left(M^{\sigma}-\eta^{\sigma}, M^{\bar{\sigma}}\right)\right) .
\end{aligned}
$$

The derivation of Eq. (32) concerns the exact exchangecorrelation energy functional of the 1-RDM. Since only approximations are available, the question is whether Eq. (32) is still useful. This question is the main subject of the next section.

In Ref. [23], a single, spin-independent $\mu$ (for closed-shell systems) was shown to have a discontinuity as a function of a fractional total number of electrons which is equally distributed in the two spin channels. The application of that theory to an open-shell system would give the spin resolved $\mu^{\sigma}$ as a function of a unique $M$. In the present work, we add (subtract) a fractional part of an electron to (from) a specific spin channel. Consequently, the system becomes an openshell system even if the neutral system is closed shell. Thus, we have four functions $\mu^{\uparrow}\left(M^{\uparrow}, N^{\downarrow}\right), \mu^{\downarrow}\left(N^{\uparrow}, M^{\downarrow}\right), \mu^{\uparrow}\left(N^{\uparrow}, M^{\downarrow}\right)$, and $\mu^{\downarrow}\left(M^{\uparrow}, N^{\downarrow}\right)$, where $N^{\uparrow}, N^{\downarrow}$ are fixed to the integer values of the neutral system. Of these four, only the first two show a discontinuity. The correct gap is then given by Eq. (32), where the min and max functions take care of the selection of the smallest $I^{\sigma}$ and the largest $A^{\sigma}$. Alternatively, one can employ Eqs. (1)-(3) for the calculation of the fundamental gap. Both approaches are exact, in the sense that, given the exact functional of $\gamma$, they both reproduce the fundamental gap. It is interesting to see if they give the same numbers for approximate functionals as well. This is also one of the questions we address in the next section.

To answer the above questions, one needs to minimize the approximate functionals for fractional number of particles to get $\mu^{\uparrow, \downarrow}\left(M^{\sigma}, N^{\bar{\sigma}}\right)$. The extension of the minimization procedure to fractional particle numbers, which is in complete accordance with the proof we presented above, requires us to perform the minimization in the domain of $\gamma_{N^{\sigma}+\eta^{\sigma}, N^{\bar{\sigma}}}^{\sigma_{1}}$, which are given by Eq. (18). In principle, one then has to minimize the total energy with respect to $\gamma_{N^{\sigma}, N^{\bar{\sigma}}}^{\sigma_{1}}$ and $\gamma_{N^{\sigma_{+}+1, N} N^{\bar{\sigma}}}^{\sigma_{1}}$ under the known $N$-representability constraints that their occupation numbers are between 0 and 1 and sum up to the correct particle numbers. However, this procedure, involving the density matrices for $N$ and $N+1$ particles, is not very practical. On the contrary, it is desirable to minimize with respect to $\gamma_{N^{\sigma_{+}} \eta^{\sigma}, N^{\bar{\sigma}}}^{\sigma_{1}}$ directly under the appropriate constraints. We prove elsewhere [29] that the appropriate constraints for such a minimization are
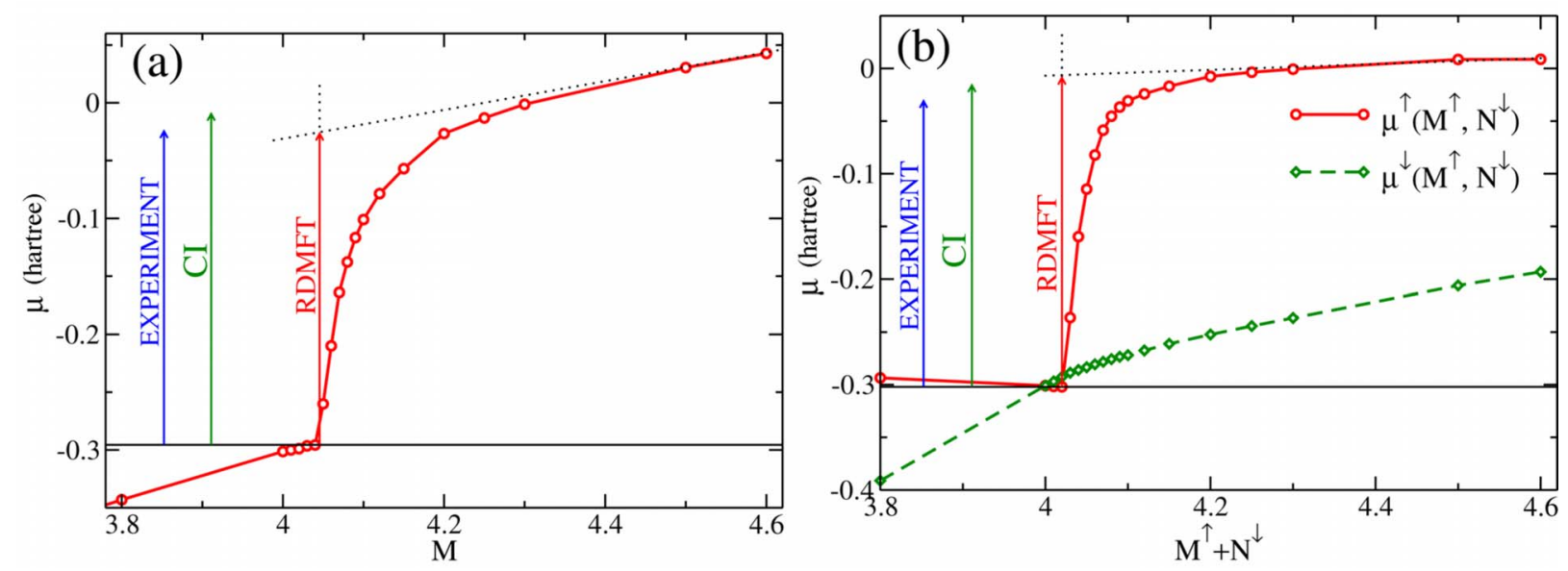

FIG. 1. (Color online) Behavior of $\mu$ as a function of a fractional electron number $M$ for the LiH molecule in a closed-shell treatment (a) and $\mu^{\uparrow, \downarrow}\left(M^{\uparrow}, N^{\downarrow}\right)$ for an open-shell treatment (b). For comparison, the experimental and configuration interaction (CI) values (see Table I) of the fundamental gap are included. 


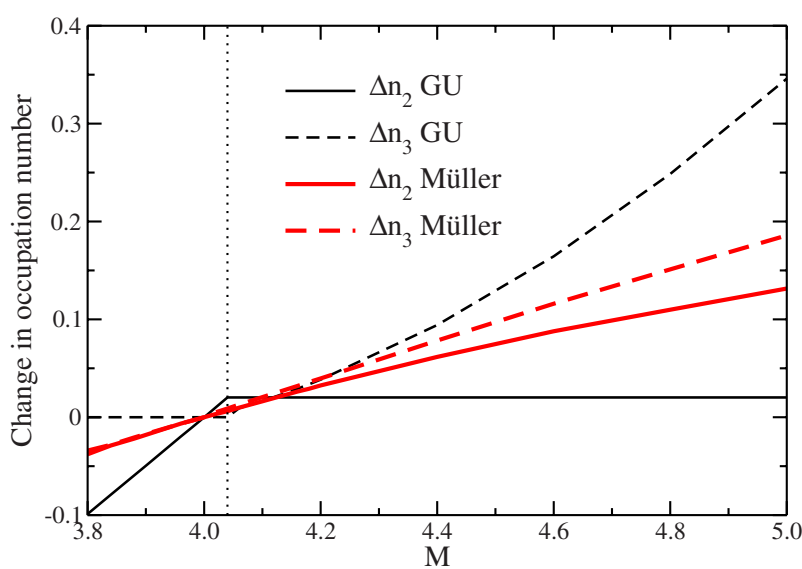

FIG. 2. (Color online) Change $\Delta n_{j}$ in the second and third occupation numbers for a closed-shell treatment of the $\mathrm{LiH}$ molecule. For the neutral system $n_{1}=1, n_{2}=0.98(0.79), n_{3}=3 \times 10^{-5}(0.09)$ for the GU (Müller) functional. For the GU functional $n_{2}$ gets pinned at $M \approx 4.05$.

$$
0 \leqslant n_{j \sigma_{1}}^{\left(M^{\sigma}, M^{\bar{\sigma}}\right)} \leqslant 1, \quad \forall j, \quad \sum_{j} n_{j \sigma_{1}}^{\left(M^{\sigma}, M^{\bar{\sigma}}\right)}=M^{\sigma_{1}} .
$$

In other words, the domain of $\gamma_{N^{\sigma}+\eta^{\sigma}, N^{\bar{\sigma}}}^{\sigma_{1}}$ which can be represented as the weighted average Eq. (18) is identical to the domain of $\gamma_{N^{\sigma}+\eta^{\sigma}, N^{\bar{\sigma}}}^{\sigma_{1}}$ whose eigenvalues satisfy Eq. (33). The above statement is quite significant since the constraint of Eq. (33) is much simpler and completely analogous to the case of integer particle numbers. The implementation is, therefore, a rather simple extension of the case of integer particle numbers.

\section{NUMERICAL RESULTS}

In this section, we study the behavior of $\mu$ as a function of the fractional particle number for some atoms and molecules using approximate functionals of the 1-RDM. Our aim is to investigate whether there exists a discontinuity in $\mu(M)$ and how it compares to the fundamental gap. The implementation we use for finite systems can be applied to both closedand open-shell [24] configurations. Some results for closedshell systems were presented in Ref. [23]. Here, we give an extended analysis for both closed- and open-shell systems.

For the open-shell treatment, we use the extension of the functional of Goedecker-Umrigar [5] (GU) described in Ref. [24]. We also investigate whether other functionals reproduce a discontinuity in a closed-shell treatment. For this purpose, we consider the functionals of Piris [8,9], where the self-interaction (SI) terms are explicitly removed, and the Müller [4] functional and the most recent BBC (from corrections to Buijse-Baerends) functionals of Gritsenko et al. [7] which contain self-interaction terms.

The implementation is based on the GAMESS program [30] which we use for the calculation of the one- and two-electron integrals. The minimization with respect to the occupation numbers and natural orbitals is then performed using the conjugate gradient method. Our program treats both closed as well as open-shell systems using the restricted open-shell
TABLE I. The prediction for the fundamental gap (in hartree) for several atoms and small molecules using the size of the step of $\mu(M)$, and a direct calculation through Eqs. (1)-(3) for the GU functional compared with experimental and other theoretical values. For the direct application of Eqs. (1)-(3), the total energies of the positive and negative ions were calculated.

\begin{tabular}{lcccc}
\hline \hline System & $\begin{array}{c}\text { RDMFT } \\
\mu(M) \text { step }\end{array}$ & $\begin{array}{c}\text { RDMFT } \\
\text { Eqs. (1)-(3) }\end{array}$ & $\begin{array}{c}\text { Other } \\
\text { theoretical }\end{array}$ & Experiment \\
\hline $\mathrm{Li}$ & 0.18 & 0.202 & $0.175^{\mathrm{a}}$ & $0.175^{\mathrm{b}}$ \\
$\mathrm{Na}$ & 0.18 & 0.198 & $0.169^{\mathrm{c}}$ & $0.169^{\mathrm{b}}$ \\
$\mathrm{F}$ & 0.54 & 0.549 & & $0.514^{\mathrm{b}}$ \\
$\mathrm{LiH}$ & $0.27{ }^{\mathrm{d}} 0.29^{\mathrm{e}}$ & 0.271 & $0.286^{\mathrm{f}}$ & $0.271^{\mathrm{g}}$ \\
\hline \hline
\end{tabular}

${ }^{\mathrm{a} Q u a d r a t i c ~ c o n f i g u r a t i o n ~ i n t e r a c t i o n ~ f r o m ~ R e f . ~[31] . ~}$

${ }^{\mathrm{b}}$ From Ref. [32].

${ }^{\mathrm{c}}$ Ionization potential from [31], electron affinity from [33].

${ }^{\mathrm{d}}$ Closed-shell treatment.

e Open-shell treatment.

${ }^{\mathrm{f}}$ Configuration interaction with singles and doubles, using the same basis set as in RDMFT.

${ }^{\mathrm{g}}$ Ionization potential from [34], electron affinity from [35].

RDMFT [24]. In short, we assume spin-dependent occupation numbers (and chemical potentials) but spin-independent natural orbitals. In that way, our method is in complete analogy to spin-restricted open-shell Hartree-Fock.

In Fig. 1(a), we show $\mu(M)$ for the $\mathrm{LiH}$ molecule using the GU functional in the closed-shell treatment, i.e., the extra charge is equally distributed over the two spin channels. Figure 1(b) shows $\mu^{\uparrow}\left(M^{\uparrow}, N^{\downarrow}\right)$ and $\mu^{\downarrow}\left(M^{\uparrow}, N^{\downarrow}\right)$ for the open-shell treatment of the $\mathrm{LiH}$ molecule, using again the $\mathrm{GU}$ functional. In the open-shell treatment, the additional charge is exclusively added to one spin channel, and here we choose the spin-up channel. Clearly, $\mu(M)$ in Fig. 1(a) and $\mu^{\uparrow}\left(M^{\uparrow}, N^{\downarrow}\right)$ in Fig. 1(b) show a pronounced step which resembles the discontinuity that one expects for the exact functional. This step has two important features: the first is that it occurs not exactly at $M=4$, i.e., the exact, integer number of electrons. It is rather shifted slightly to the right. The shift is of the order of 0.05 of an electron in Fig. 1(a) and is reduced to 0.02 in Fig. 1(b). In Fig. 2, we plot the change in the occupation numbers (compared to their values at $N=4$ ) as a function of the total number of electrons for the closed-shell treatment. Comparison of Figs. 1(a) and 2 reveals that the bottom of the step for the GU functional appears exactly at the point where the largest fractional occupation number becomes equal to 1 . After that point it has to remain 1 , due to the $N$-representability constraints, Eq. (33). The occupation number $n_{3}$ is almost constant for $M<4.05$ and increases significantly after $n_{2}$ gets pinned. We can also see that no pinning occurs for the Müller functional where no step is observed, as we discuss later. The pinning of the largest fractional occupation number to 1 results in the rapid increase of $\mu$. Since adding charge to one spin channel only results in faster pinning of this state, it is not surprising that the step in the open-shell treatment is shifted to the left. Upon increasing the extra charge further, $\mu$ is a smooth function, i.e., the upper edge of the step is rounded off. In the closed-shell treatment, $\mu(M)$ shows a linear dependence out- 


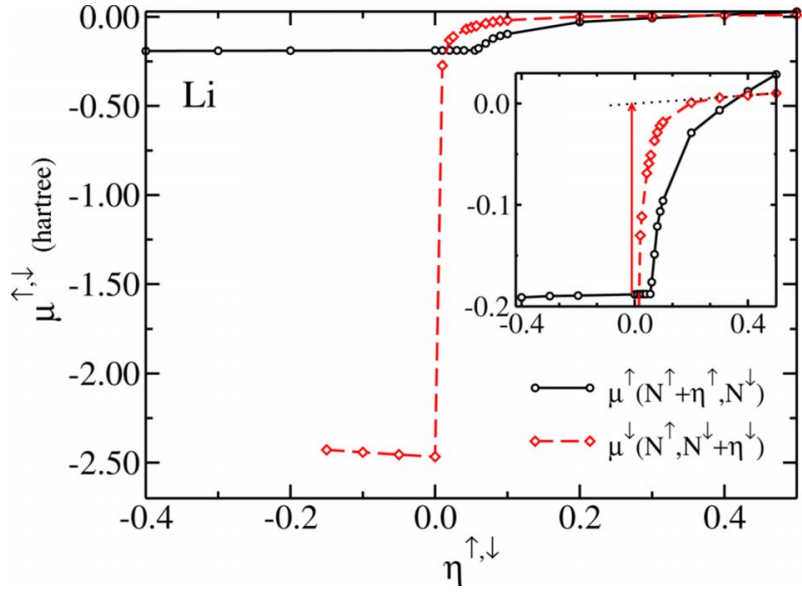

FIG. 3. (Color online) Behavior of $\mu^{\sigma}$ as a function of an electron fraction $\eta^{\sigma}$ added to (subtracted from) the neutral system for the $\mathrm{Li}$ atom. In the inset, we show an enlargement of the region where we extract the value for the gap from the difference of the upper level of $\mu^{\downarrow}\left(N^{\uparrow}, M^{\downarrow}\right)$ and the lower level of $\mu^{\uparrow}\left(M^{\uparrow}, N^{\downarrow}\right)$.

side the step region, which is significantly reduced in $\mu^{\uparrow}$ in closer resemblance to the exact behavior. A more detailed investigation reveals that the slope of $\mu(M)$ is the average of the slopes of $\mu^{\uparrow}\left(M^{\uparrow}, N^{\downarrow}\right)$ and $\mu^{\downarrow}\left(M^{\uparrow}, N^{\downarrow}\right)$. To extract a value for the discontinuity, we use a backward projection as shown in Fig. 1. This method reduces to the exact discontinuity if $\mu$ is a true step function. The extracted values, as well as the gaps of other finite systems are given in Table I. We should also keep in mind that DFT methods, like the LDA and GGA, underestimate the gap by typically $50 \%$. Although the procedure of backward projection might seem rather crude and arbitrary, we should mention that the agreement with experiment is rather satisfactory for both closed- and openshell treatments. As one can see, for $\mathrm{LiH}$, the quantitative agreement is slightly better for a closed-shell treatment. Nevertheless, the open-shell treatment should be preferred because $\mu$ then resembles the exact step function much closer, making the backward projection less ambiguous.

For open-shell systems, varying $M^{\uparrow}$ or $M^{\downarrow}$ is no longer equivalent. Thus, we can study the behavior of both $\mu^{\uparrow}$ and $\mu^{\downarrow}$ as functions of $M^{\uparrow}$ or $M^{\downarrow}$. We investigate the open-shell atoms $\mathrm{Li}, \mathrm{Na}$, and $\mathrm{F}$, varying $M^{\uparrow}$ or $M^{\downarrow}$ away from the neutral configurations. In the following, we use the convention that spin up is always the majority spin channel. In Fig. 3, we show the results for $\mu^{\sigma}$ for the Li atom. Only the chemical potential corresponding to the spin direction whose particle number is changed shows a discontinuity as already observed for the $\mathrm{LiH}$ molecule. Therefore, we plot only $\mu^{\uparrow}\left(M^{\uparrow}, N^{\downarrow}\right)$ and $\mu^{\downarrow}\left(N^{\uparrow}, M^{\downarrow}\right)$. Again, pronounced steps resembling the discontinuity of the exact theory are present. The prediction for the gap is then selected using Eq. (32) and the backward extrapolation procedure described earlier. The values obtained for the gaps are listed in Table I. According to Eq. (32), the gap for the $\mathrm{Li}$ atom is given by the difference between the backward-projected upper part of $\mu^{\downarrow}\left(N^{\uparrow}, M^{\downarrow}\right)$ and the lower part of $\mu^{\uparrow}\left(M^{\uparrow}, N^{\downarrow}\right)$. In Fig. 4, we show the analogous results for the $\mathrm{Na}$ and $\mathrm{F}$ atoms. The picture for the $\mathrm{Na}$ atom is very similar to Li. On the other hand, for the $\mathrm{F}$ atom, the gap is given by $\mu^{\downarrow}\left(N^{\uparrow}, M^{\downarrow}\right)$ alone. It is interesting that the position of the upper and lower parts of the $\mu^{\sigma}$ corresponds to the actual process of adding (removing) electrons to (from) the system. Thus, for $\mathrm{Li}$ and $\mathrm{Na}$ atoms, it is favorable to remove an electron from the majority spin channel (up) and add an extra electron to the minority spin channel (down). As a consequence, the gap is given by the difference between the upper part of $\mu^{\downarrow}\left(N^{\uparrow}, M^{\downarrow}\right)$ and the lower part of $\mu^{\uparrow}\left(M^{\uparrow}, N^{\downarrow}\right)$. For a $\mathrm{F}$ atom, on the other hand, it is favorable to add an electron to, or remove from, the minority spin channel. Thus, the gap is given by $\mu^{\downarrow}\left(N^{\uparrow}, M^{\downarrow}\right)$ alone.

In Table I, we give the results obtained by the backward extrapolation for the systems discussed in this paper. As one can see, they agree very well with experimental values for the fundamental gap as well as other theoretical calculations. For finite systems, one can also calculate the gap by performing three total energy calculations, for the $N$-, the $(N+1)$-, and $(N-1)$-particle systems and use Eqs. (1)-(3). The values for the gap obtained in this way are given in Table I for comparison. One should keep in mind that, for solid state systems, this procedure does not apply because the addition or the removal of a single electron in an infinite solid is meaningless. For such systems, the recipe introduced in this work is expected to be valuable [36].
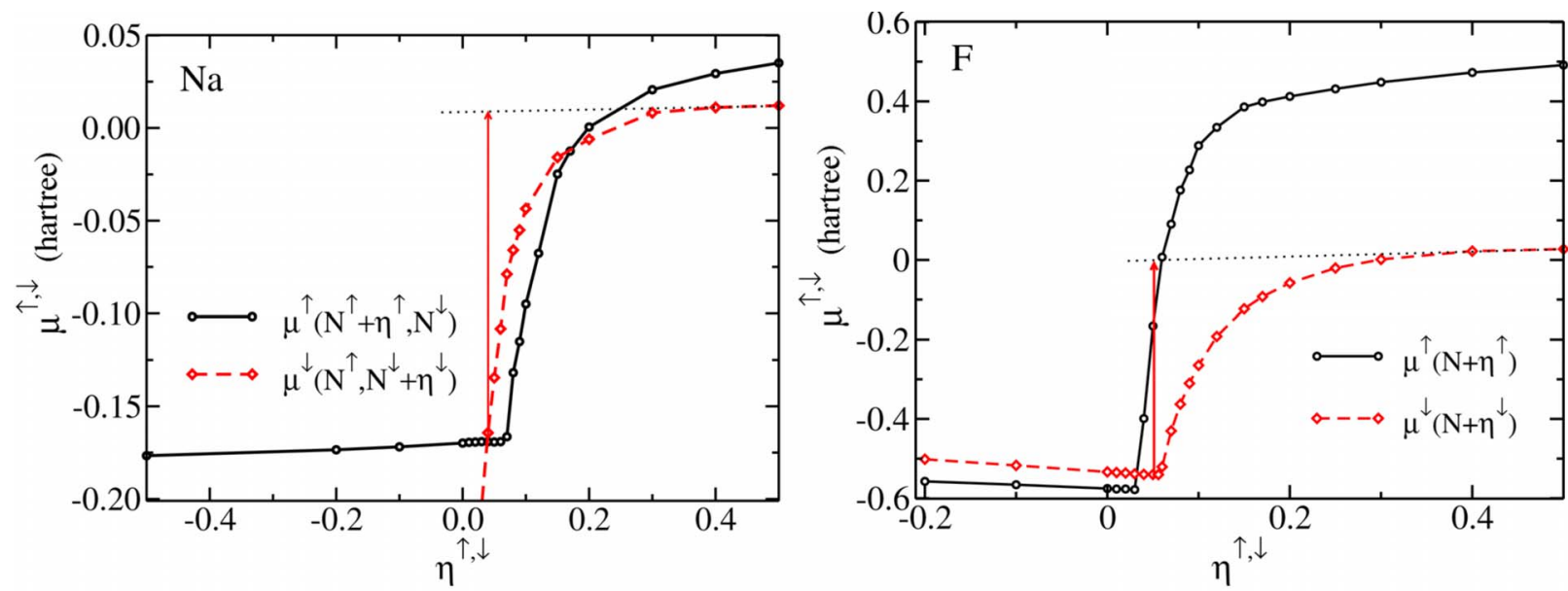

FIG. 4. (Color online) Behavior of $\mu^{\sigma}$ as a function of an electron fraction $\eta^{\sigma}$ added to (subtracted from) the neutral system for Na and $\mathrm{F}$ atoms. For $\mathrm{Na}$, we show only the regions from which the values of the gap are extracted. 


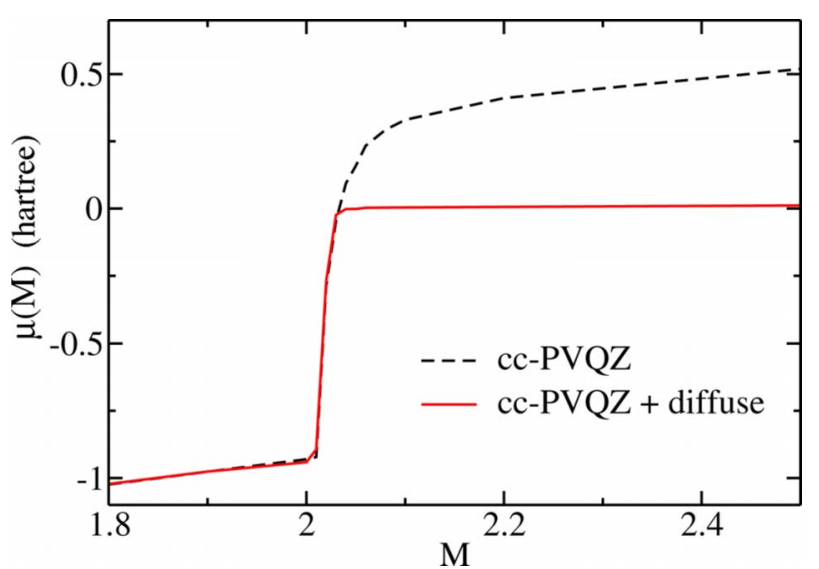

FIG. 5. (Color online) Function $\mu(M)$ for the He atom using the cc-PVQZ basis set without and with an additional very diffuse $s$-type basis function.

Of course, the question arises whether the system with excess charge is correctly described by the basis set we used. Usually, atomic basis sets are optimized to correctly describe the neutral system, resulting in basis functions which are all localized. Therefore, the charged system might be predicted to have a localized bound state despite the fact that the configuration of a neutral atom and a free completely delocalized electron is energetically favorable. A prominent example of a system not having a negative ion is the He atom. We study the behavior of $\mu(M)$ with two different basis sets: the correlation consistent polarized valence quadruple zeta (ccPVQZ) basis set and cc-PVQZ enlarged by a very diffuse $s$-type function. As one can see in Fig. 5, the state of the additional fractional electron is better described by the enlarged basis set. In this case, the electron affinity is zero and the gap is given by the ionization potential alone. Interestingly, the inclusion of a diffuse function leads to a sharper step of $\mu(M)$ in close resemblance to the discontinuity of the exact functional. We also add extra diffuse functions in the basis sets of both $\mathrm{Li}$ and $\mathrm{H}$ in the calculation of $\mu(M)$ for the $\mathrm{LiH}$ molecule. We do not observe any effect on $\mu(M)$, which is clear evidence for the fact that $\mathrm{LiH}$ binds an extra electron and that the localized basis set is appropriate for describing the state of the charged system.

In order to investigate the importance of the variation of the natural orbitals for the discontinuity of $\mu$, we perform, apart from the full variation described so far, a calculation where only the occupation numbers are optimized, while for the natural orbitals we keep the initial Hartree-Fock orbitals. In Fig. 6, we compare these two procedures for both a closed- and an open-shell calculation. As one can see from the plots, the main contribution to the discontinuity arises from the variation of the occupation numbers. In the closedshell calculation, we obtain a discontinuity of 0.27 hartree for the full variation, compared to 0.26 hartree if we vary the occupation numbers only. In other words, only about $4 \%$ of the discontinuity is due to the optimization of the natural orbitals. This picture remains unchanged if we use the openshell procedure, where we obtain 0.31 hartree for the full variation and 0.29 hartree for the variation of the occupation numbers alone.

In all the calculations presented so far, we have used the functional of Goedecker and Umrigar, which involves the complete removal of the self-interaction terms. It is interesting to study the behavior of $\mu(M)$ using different functionals, like, for instance, the recent BBC functionals of Gritsenko et al. [7] and the Piris natural orbital functional (PNOF) of Piris [8,9]. In the BBC1 and BBC2 functionals, the SI terms are present while in the $\mathrm{BBC} 3$, they are partially removed. However, the SI terms for the bonding and the antibonding orbitals remain. In the PNOF functional, they are fully removed, as in the GU one. In Fig. 7, we plot $\mu(M)$ for $\mathrm{LiH}$ using the closed-shell treatment, for all these functionals. In consistence with earlier findings [23], only the GU and PNOF functionals show a pronounced step, which compares well with the fundamental gap. The other functionals show either a completely smooth behavior or, in the case of BBC3, a small kink in the wrong direction. Therefore, we conclude that the complete removal of the SI terms is essential for obtaining the correct behavior of $\mu(M)$. The size of the step of $\mu(M)$ for the PNOF functional is 0.30 hartree and compares well with experiment (see Table I). As a test, we also tried a modified version of $\mathrm{BBC} 3$, where we removed
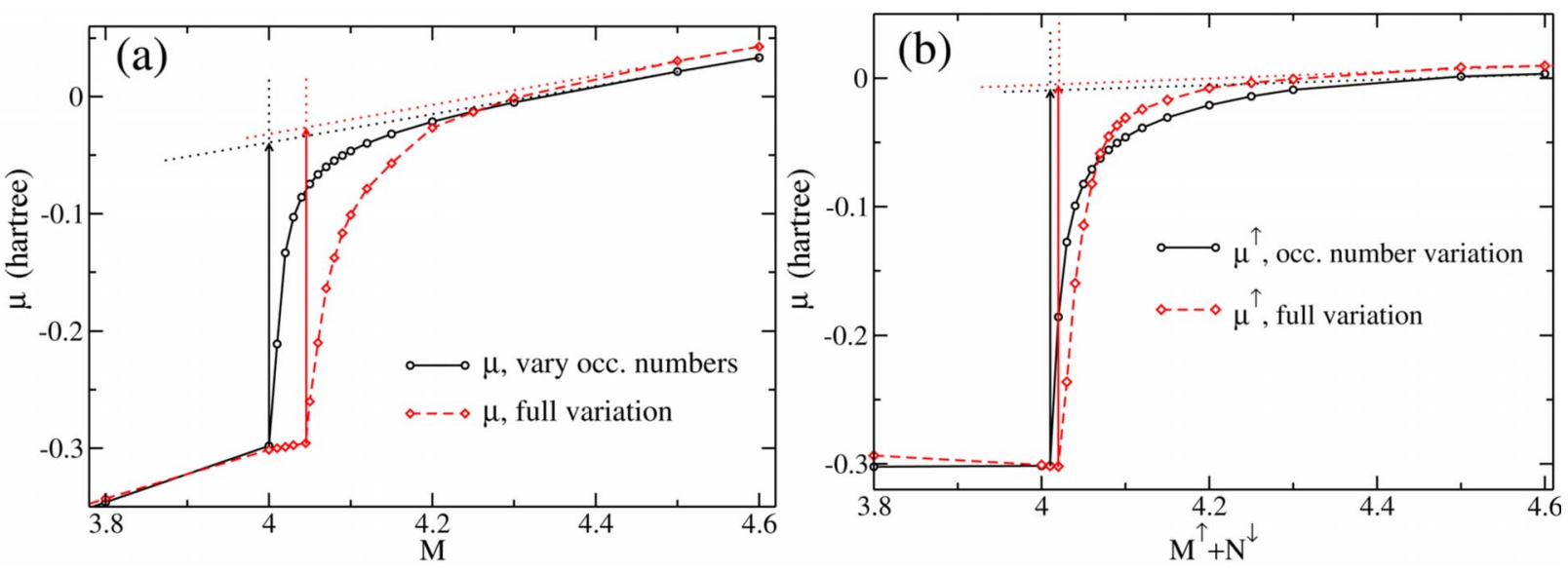

FIG. 6. (Color online) Function $\mu(M)\left[\mu^{\uparrow}\left(M^{\uparrow}, N^{\downarrow}\right)\right]$ for the LiH molecule using the closed-shell (a) and the open-shell treatment (b), with occupation number variation (using the Hartree-Fock orbitals) and with full variation (both occupation numbers and the orbitals). 


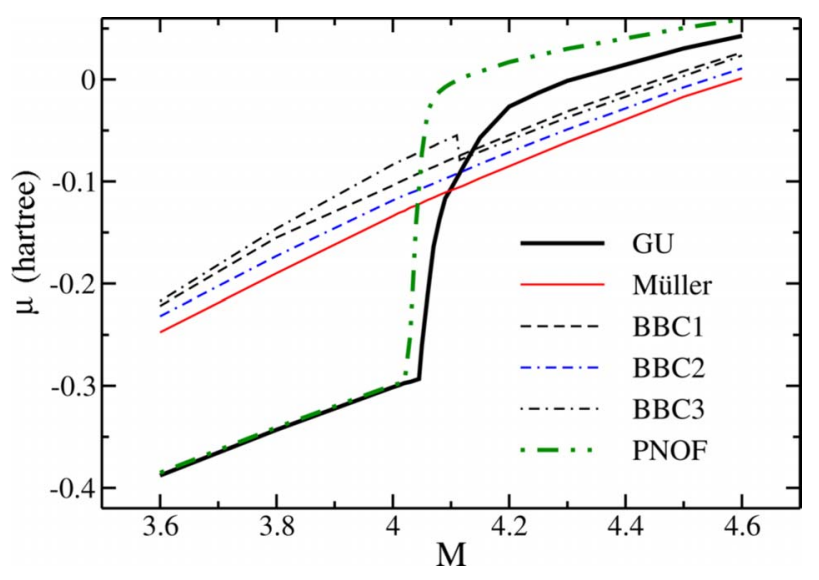

FIG. 7. (Color online) Function $\mu(M)$ for the $\mathrm{LiH}$ molecule using the closed-shell treatment for the Goedecker-Umrigar, Müller, $\mathrm{BBC} 1, \mathrm{BBC} 2, \mathrm{BBC} 3$, and PNOF functionals. The first and the last involve a complete removal of the SI terms. Only these two reproduce a pronounced step in resemblance to the discontinuity of the exact theory.

the SI terms completely. Consistent with the above conclusion, it also produces a step which is almost identical to that of PNOF. Additionally, this modified BBC3, like the GU functional, gives an accurate measure of the correlation energy at the equilibrium distance, but fails completely at the dissociation limit.

\section{CONCLUSION}

We have presented a formalism to calculate the fundamental gap within RDMFT for both open as well as closedshell systems. Our numerical results show that, even for systems where the neutral system is a closed shell, the results for the chemical potential are closer to the exact step function if an open-shell treatment is employed because addition of the charge of a specific spin to the system makes it open shell. The application to several open-shell systems gives a very good agreement with experimental values in all cases. Also, the steps in the chemical potentials are such that they resemble the spin dependence of the ionization potential and the electron affinity of the real system. Our investigation of a possible basis set dependence reveals that it is necessary to include very diffuse states in the basis set in case the system does not bind extra charge. Whenever the system does bind extra charge, the results are independent of the inclusion of the diffuse state in the basis set. To estimate the contribution of the occupation numbers and the natural orbitals to the fundamental gap we compared the results for the $\mathrm{LiH}$ molecule using a full variation and a variation of the occupation numbers only. We found that over $90 \%$ of the fundamental gap is due to the occupation numbers. This finding was confirmed for several other systems so far, and we believe that it shows a general feature of RDMFT calculations. Finally, we investigated the behavior of several different functionals for the calculation of the fundamental gap. From our results we conclude that the exclusion of the self-interaction for all natural orbitals is essential to obtain reasonable results. Functionals without any removal of self-interaction simply yield a continuous chemical potential.

The present work is a contribution to the subject of calculating the fundamental gap of materials within RDMFT. The hope is that this theory gives results closer to experiment than does DFT for this fundamental problem. It is our belief that the theoretical development presented in this work will have a significant impact in the application of RDMFT to periodic systems.

\section{ACKNOWLEDGMENTS}

We would like to thank A. Zacarias for valuable discussions on experimental and different theoretical results. This work was supported in part by the Deutsche Forschungsgemeinschaft within the program SPP 1145, and by EU's Sixth Framework Program through the Nanoquanta Network of Excellence (Grant No. NMP4-CT-2004-500198).
[1] P. Hohenberg and W. Kohn, Phys. Rev. 136, B864 (1964).

[2] W. Kohn and L. Sham, Phys. Rev. 140, A1133 (1965).

[3] T. Gilbert, Phys. Rev. B 12, 2111 (1975).

[4] A. Müller, Phys. Lett. 105A, 446 (1984).

[5] S. Goedecker and C. J. Umrigar, Phys. Rev. Lett. 81, 866 (1998).

[6] M. Buijse and E. J. Baerends, Mol. Phys. 100, 401 (2002).

[7] O. Gritsenko, K. Pernal, and E. J. Baerends, J. Chem. Phys. 122, 204102 (2005).

[8] M. Piris, Int. J. Quantum Chem. 106, 1093 (2006).

[9] P. Leiva and M. Piris, J. Chem. Phys. 123, 214102 (2005).

[10] M. A. L. Marques and N. N. Lathiotakis, Phys. Rev. A 77, 032509 (2008).

[11] N. N. Lathiotakis and M. A. L. Marques, J. Chem. Phys. 128, 184103 (2008).

[12] N. N. Lathiotakis, N. Helbig, and E. K. U. Gross, Phys. Rev. B
75, 195120 (2007).

[13] J. P. Perdew and M. Levy, Phys. Rev. Lett. 51, 1884 (1983).

[14] L. J. Sham and M. Schlüter, Phys. Rev. B 32, 3883 (1985).

[15] M. Städele, J. A. Majewski, P. Vogl, and A. Görling, Phys. Rev. Lett. 79, 2089 (1997).

[16] M. Städele, M. Moukara, J. A. Majewski, P. Vogl, and A. Görling, Phys. Rev. B 59, 10031 (1999).

[17] S. Sharma, J. K. Dewhurst, and C. Ambrosch-Draxl, Phys. Rev. Lett. 95, 136402 (2005).

[18] M. Grüning, A. Marini, and A. Rubio, J. Chem. Phys. 124, 154108 (2006).

[19] J. Heyd, G. E. Scuseria, and M. Ernzerhof, J. Chem. Phys. 118, 8207 (2003).

[20] J. Heyd and G. E. Scuseria, J. Chem. Phys. 120, 7274 (2004).

[21] J. Heyd, J. E. Peralta, G. E. Scuseria, and R. L. Martin, J. Chem. Phys. 123, 174101 (2005). 
[22] J. P. Perdew, R. G. Parr, M. Levy, and J. L. Balduz, Jr., Phys. Rev. Lett. 49, 1691 (1982).

[23] N. Helbig, N. N. Lathiotakis, M. Albrecht, and E. K. U. Gross, Europhys. Lett. 77, 67003 (2007).

[24] N. N. Lathiotakis, N. Helbig, and E. K. U. Gross, Phys. Rev. A 72, 030501(R) (2005).

[25] P. Leiva and M. Piris, Int. J. Quantum Chem. 107, 1 (2007).

[26] L. J. Sham and M. Schlüter, Phys. Rev. Lett. 51, 1888 (1983).

[27] W. Kohn, Phys. Rev. B 33, 4331 (1986).

[28] A. Coleman, Rev. Mod. Phys. 35, 668 (1963).

[29] S. Sharma et al. (unpublished).

[30] M. W. Schmidt et al., J. Comput. Chem. 14, 1347 (1993).
[31] J. A. Montgomery, Jr., J. W. Ochterski, and G. A. Petersson, J. Chem. Phys. 101, 5900 (1994).

[32] A. A. Radzig and B. M. Smirnov, Reference Data on Atoms and Molecules (Springer-Verlag, Berlin, 1985).

[33] J. J. De Groote and M. Masili, J. Chem. Phys. 120, 2767 (2004).

[34] H. R. Ihle and C. H. Wu, J. Chem. Phys. 63, 1605 (1975).

[35] S. B. Sharp and G. I. Gellene, J. Chem. Phys. 113, 6122 (2000).

[36] S. Sharma, J. K. Dewhurst, N. N. Lathiotakis, and E. K. U. Gross, Phys. Rev. B 78, 201103(R) (2008). 